\title{
Formation of massive stars through disk accretion $\dagger$
}

\author{
Rolf Chini $^{1}$, V. H. Hoffmeister ${ }^{1}$, M. Nielbock ${ }^{1}$, C. M. Scheyda ${ }^{1}$, \\ D. Nürnberger ${ }^{2}$, \\ E. D. Feigelson ${ }^{3}$, K. Getman ${ }^{3}$ and L. K. Townsley ${ }^{3}$ \\ ${ }^{1}$ Astronomical Institute, Ruhr-Universität Bochum, 44780 Bochum, Germany \\ email: chini@astro.rub.de \\ ${ }^{2}$ European Southern Observatory, Casilla 19001, Santiago 19, Chile \\ ${ }^{3}$ Dept. of Astronomy and Astrophysics, Pennsylvania State University, PA 16802, USA
}

\begin{abstract}
IR imaging, $K$-band spectroscopy and $X$-ray data of the stellar cluster in M 17 suggests three categories of massive young objects: I) "Naked" early-type O-stars in the cluster center that exhibit $X$-ray emission and normal optical/IR-spectra; they do not show signs for any circumstellar material. II) Late O-type stars, that show $X$-ray emission and $\mathrm{H} / \mathrm{He}$ absorption lines in their optical/IR-spectra. There is evidence for extended circumstellar material in the form of elongated $L$-band nebulae, circumstellar MIR emission from regions of about $10.000 \mathrm{AU}$ in diameter and filaments of He I $1.083 \mu \mathrm{m}$. III) Massive stars without $X$-ray emission, $K$-band spectra with emission lines of $\mathrm{H}$ and $\mathrm{He}$ and in some cases with $\mathrm{CO}$ band head emission. They are heavily enshrouded and also show elongated $L$-band nebulae, circumstellar MIR emission from regions of about 4.000 AU and ionized He envelopes.

Obviously, M 17 harbors several generations of massive stars: Only the oldest "Category I", located in the cluster center, is free of circumstellar material. "Category II" and "III" comprise younger dust-enshrouded objects of a second generation embedded in the interface of the HII region and the adjacent molecular cloud. Due to the presence of flattened dusty disks and perpendicular gaseous outflows, accretion seems to be important for the formation of stars up to type $\mathrm{O} 8$.

A population of X-ray emitting sources with $\left(L_{\mathrm{bol}} \sim 10^{4} \mathrm{~L}_{\odot}\right)$ shows $\mathrm{CO}$ band head absorption; one source was found to have variable $\mathrm{CO}$ absorption as known from FU Orionis stars.

New data on the 20.000 AU accretion disk in M 17 reveals a collimated $\mathrm{H}_{2} 2.122 \mu \mathrm{m}$ jet with three distinct emission knots that emerges from the disk center. The broad $\mathrm{H} \alpha$ and Ca II emission lines from the bipolar nebula display blue-shifted absorption originating from accretion disk-driven outflows with velocities of $\pm 500 \mathrm{~km} / \mathrm{s}$.
\end{abstract}

Keywords. stars: formation, stars: pre-main-sequence, ISM: jets and outflows, X-rays: stars

\section{Introduction}

M17, one of the most luminous galactic HII regions, harbors an extremely young embedded cluster. It is a treasure box to investigate the formation of massive stars because it shows all signs of the recent creation of a new generation of O-type stars. As such it is ideal to search for evidence of circumstellar material still associated with massive protostellar objects and to answer the question whether disk accretion plays the major role in forming also the most massive stars.

Our full data set on the M17 cluster comprises BVRI imaging with EMMI at the NTT (Schmidt et al., in prep.), JHK imaging with ISAAC at the VLT (Hoffmeister et al., in prep.) $L$ imaging with ISAAC (Scheyda et al., in prep.), narrow-band (HeI,

$\dagger$ Based on observations made with ESO Telescopes at the La Silla and Paranal Observatories 
FeI, $\mathrm{H}_{2}$ ) imaging with SOFI (Nielbock et al., in prep.), $K$-band spectroscopy with ISAAC (Hoffmeister et al., in prep.) and Chandra data (Townsley et al. (2003). Here we concentrate on the most massive stars and show some characteristic results for a few template objects. We assume that $X$-ray sources also seen in other wavebands are very likely to be cloud members.

\section{Massive stars in M17}

The investigation of ionizing sources in M 17 started with the $U B V R I$ photometry by Chini et al. (1980) (CEN) and found a preliminary end with the $K$-band spectroscopy by Hanson et al. (1997). Recent infrared studies (e.g. Chini \& Wargau 1998, Nielbock et al. 2001) revealed further massive objects deeply embedded along the interface between the HII region and the molecular cloud. They likely represent a new generation of massive stars whose formation were triggered by the existing O-stars. In the following, we present new spectroscopic and imaging data for already known O-stars and newly discovered massive protostellar candidates. Their $K$-band spectra and their circumstellar emission suggests three distinct categories.

\subsection{Category I - the "naked" O-stars}

All photometric and spectroscopic studies so far led to the identification of about a dozen early-type stars that can account for both the observed Lyman continuum flux and the total luminosity from M 17. Investigating the circumstellar environment of eight bonafide ionizing sources by means of imaging at $L, N$ and $Q$ and by narrow-band imaging in the NIR lines of $\mathrm{He}$, Fe I and $\mathrm{H}_{2}$ yields no evidence for any circumstellar material. Thus, these early type stars are "naked" and do not tell anything about their origin.
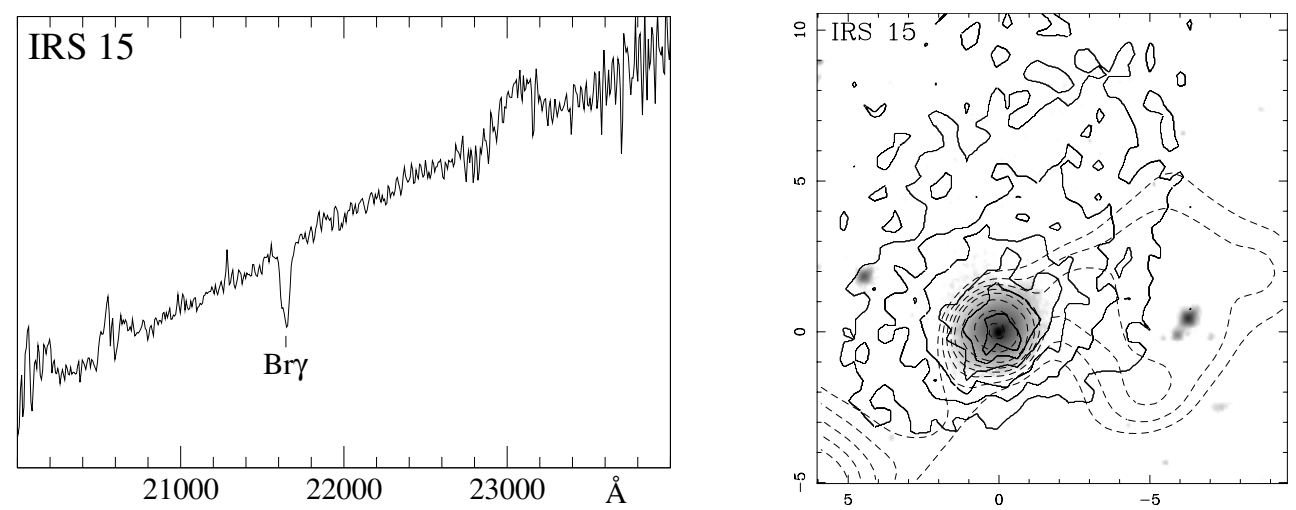

Figure 1. Left: $K$-band spectrum of IRS 15, a deeply embedded object from Category II. Right: Circumstellar environment of IRS 15 (offsets in arcsec). The gray scale shows the protostellar source at $3.7 \mu \mathrm{m}$ along with the emission of hot dust. The solid contours display the $10 \mu \mathrm{m}$ emission from warm dust in a circumstellar disk. The dashed contours represent the He I $1.083 \mu \mathrm{m}$ filamentary emission.

\subsection{Category II - O-type stars with circumstellar disks}

There are a number of optically visible stars with unique spectral designations of late O. Two examples are CEN 16 and 31, which both are Chandra sources and exhibit $K$-band spectra with prominent $\operatorname{Br} \gamma$ absorption lines. Interestingly, our $L$-band images show fan-shaped $L$-band nebulae in the vicinity of both stars. Moreover, MIR imaging reveals flattened emission regions of $11.400 \times 9.200 \mathrm{AU}$ and $16.500 \times 11.000 \mathrm{AU}(\mathrm{FWHM})$, 
respectively, corroborating the presence of huge circumstellar disks. Likewise, narrowband images in the HeI $1.083 \mu \mathrm{m}$ line show bipolar filaments of several thousand AU.

A new example for a Category II object is IRS 15, discovered by Chini \& Wargau (1998). It is a Chandra source and its $K$-band spectrum (Fig. 1) resembles those of the O-stars CEN 16 and 31; likewise, the observed dereddened luminosity from $0.3-20 \mu \mathrm{m}$ of $>3.7 \times 10^{3} \mathrm{~L}_{\odot}$ is compatible with an early type. The circumstellar environment of IRS 15 consists of an elliptical region of $L$-band emission, probably originating from hot dust close to the star, and a $10 \mu \mathrm{m}$ emission region of $11.400 \times 8.800 \mathrm{AU}$ (FWHM), most likely due to cooler dust. Like in the case of the O-stars CEN 16 and 31 there is a huge He I $1.083 \mu \mathrm{m}$ filament originating from the central source (Fig. 1).

\subsection{Category III - massive stars with circumstellar shells}

The prototype for this category of massive stars is M17 SW or M17-UC1, originally discovered by Felli et al. (1984). It is an ultra-compact HII region without X-ray emission whose elongated $K$-band morphology suggests that it might be a binary source. The strongly reddened $K$-band spectrum displays only the $\mathrm{Br} \gamma$ line in emission. Our $K$ and $L$-band images show a near-by elongated nebula; the strong circumstellar MIR emission originates from an area of $4.200 \times 3.500 \mathrm{AU}(\mathrm{FWHM})$.
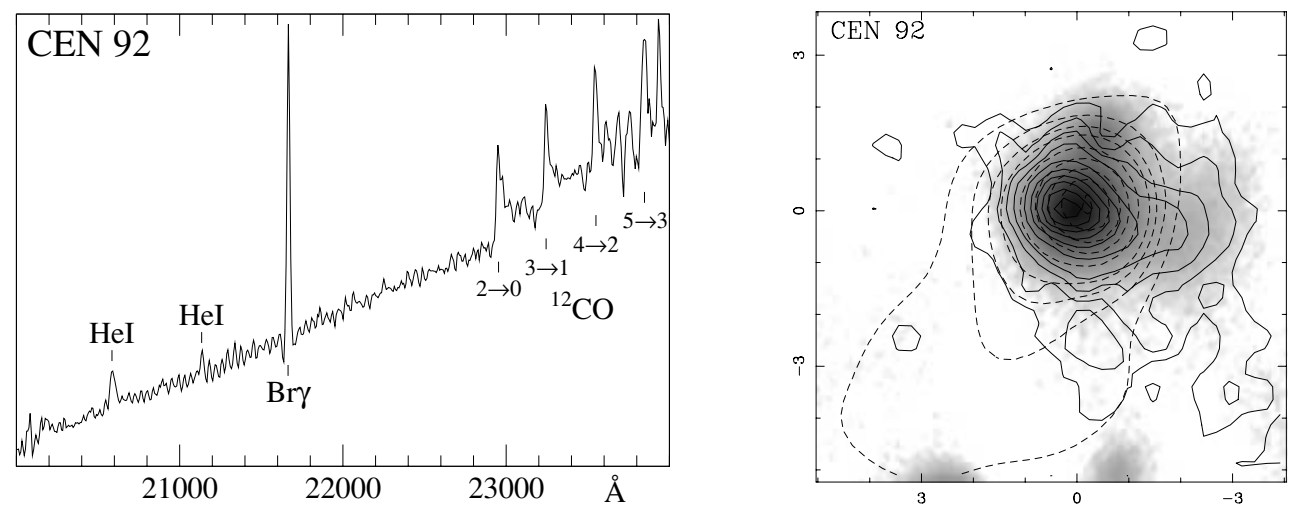

Figure 2. Left: $K$-band spectrum of CEN 92, an embedded binary object from Category III. The emission lines of $\mathrm{H}$ and He originate from the star itself and not from the nebulous environment. Strong CO band head emission indicates the presence of warm, dense circumstellar material. Right: Circumstellar environment of CEN 92 (same notation as Fig. 1.)

CEN 92 shows many similarities with M 17-UC1. It is also a binary source without X-ray emission whose reddened $K$-band spectrum displays $\mathrm{Br} \gamma$ and $\mathrm{HeI}$ in emission (Fig. 2). The observed dereddened luminosity from $0.3-20 \mu \mathrm{m}$ of $>10^{5} \mathrm{~L}_{\odot}$ is compatible with a late O-type star. There is an elongated $L$-band nebula, and circumstellar MIR emission from an area of $4.200 \times 3.100 \mathrm{AU}(\mathrm{FWHM})$. Perpendicular to the flattened dust emission there is a strong He I $1.083 \mu \mathrm{m}$ lobe.

\section{Peculiar massive objects}

While about 20 of the above objects show striking similarities that demand a classification, there are a number of deeply embedded massive sources whose nature does not fit into this scheme. 


\subsection{IRS 5 - a massive protostellar triple system}

IRS 5 - one of the strongest MIR sources in the region - has been investigated by Chini et al. (2001) and Kassis et al. (2002). It was regarded as a (single) massive protostellar object embedded in a dense dust shell. From the SED given by Nielbock et al. (2001) we derive a dereddened luminosity from $0.3-20 \mu \mathrm{m}$ of $\sim 1.2 \times 10^{5} \mathrm{~L}_{\odot}$.

Our $K$ and $L$-band images resolve IRS 5 into three objects (see Fig. 3 ) where the two fainter companions have projected distances of 2.000 and 5.000 AU from the brightest object, respectively; only the brightest component is a Chandra source. This triple system is encircled by two opposite elongated/fan-shaped $L$-band nebulae. Its common circumstellar MIR emission comes from a region of $6.200 \times 5.500 \mathrm{AU}(\mathrm{FWHM})$.

\subsection{B273 - a massive FU Orionis object?}

Located in one of the most active regions of M 17, B 273 is an infrared star which has been misidentified by Hanson et al. (1997) as the ultracompact HII region (M 17 SW) due to its immediate neighborhood to the actual UCHII region M 17-UC1. These authors present a $K$-band spectrum of B 273 (their Fig. 18) which shows strong emission lines of He I $2.058 \mu \mathrm{m}$ and $\operatorname{Br} \gamma 2.166 \mu \mathrm{m}$ and state that this emission originates exclusively from the unresolved point source. As shown by our high-resolution images (see Fig. 3), the extended nebular emission is quite strong in that region and has most likely contaminated the 1.5 arcsec slit spectrum by Hanson et al. (1997) severely. Our new spectrum of B 273 (Fig. 3) proves that this star neither has emission lines nor is the ultra-compact HII region M17-UC1.
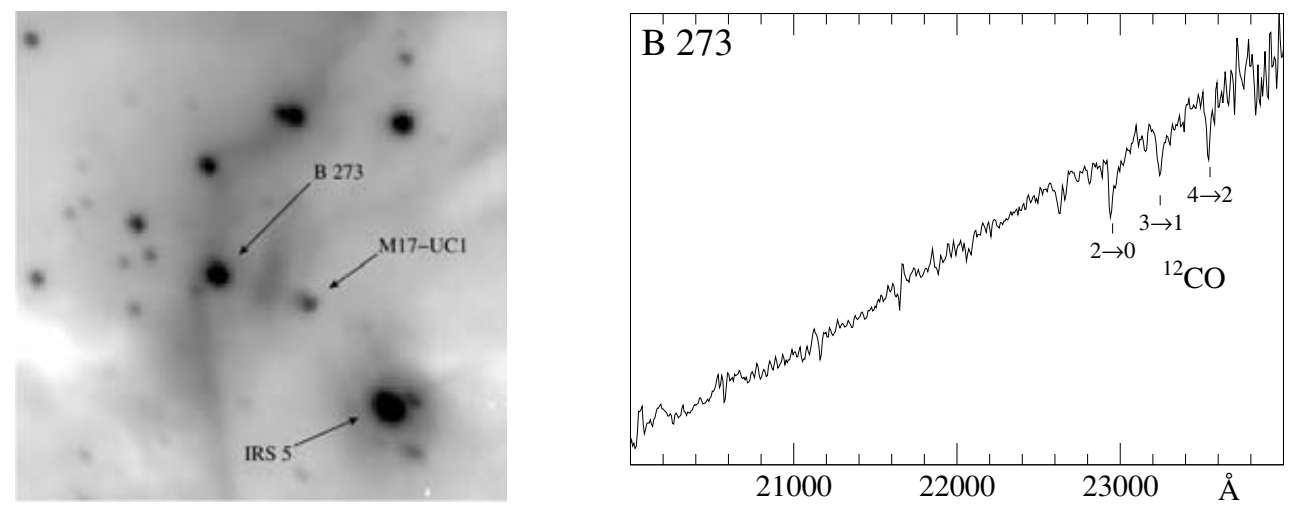

Figure 3. Left: ISAAC $K$-band image of the "Arc" region, showing the relative locations of B 273, M 17-UC1 and IRS 5. Right: $K$-band spectrum of B 273, a peculiar massive object with $\sim 10^{5} \mathrm{~L} \odot$. The absence of significant $\mathrm{H}$ and He lines indicates strong veiling by circumstellar dust. The spectrum shows clear CO band head absorption which was not detected in 1995. This suggests variability similar to that seen in FU Orionis objects.

More interesting, however, is the fact that the spectrum of B 273 shows strong CO band head absorption (Fig. 3); this was not detected by Hanson et al. (1997) during their observations in 1995. Given that B 273 is an X-ray source with an $L$-band excess and an observed luminosity of $\sim 10^{5} \mathrm{~L}_{\odot}$ we propose that it is a massive FUOR-like object with variable $\mathrm{CO}$ band head strength.

\subsection{Disk accretion in $M 17$}

The massive objects from Cat. II and III discussed above show clear evidence for circumstellar dust, most likely in the form of disks; additionally, there are gaseous flows from 
He. However, direct evidence for accretion is missing in these sources. In a recent paper, Chini et al. (2004) report the discovery of a 20.000 AU flared silhouette from a disk seen almost edge-on. Molecular line data from the $\mathrm{CO}(1-0)$ transition indicate that this object is rotating. A bipolar nebula, perpendicular to the disk suggests the presence of winds from the protostellar object which is probably the youngest massive source within the region. However, apart from an elliptical $K$-band source of $\sim 19.5$ mag there is no direct information on the protostellar source so far.
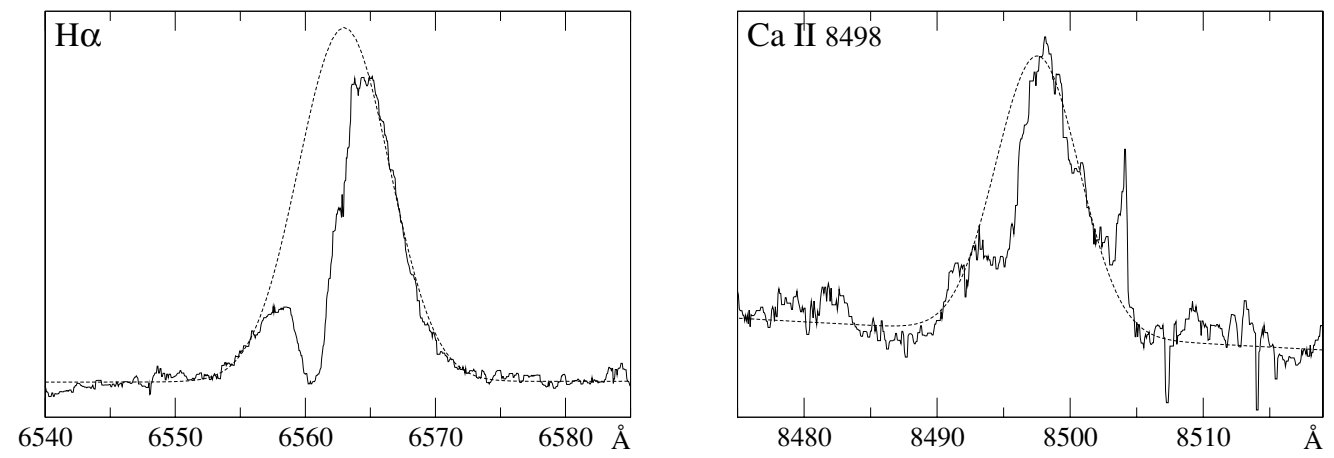

Figure 4. $\mathrm{H} \alpha$ and $\mathrm{Ca}$ II line profiles from the bipolar nebula associated with the huge disk in M 17. The blue-shifted absorption indicates accretion disk-driven outflows ( $\Delta v$ is $\pm 500 \mathrm{~km} / \mathrm{s}$.)

Our narrow-band image in the $\mathrm{H}_{2} 2.122 \mu \mathrm{m}$ line shows a collimated jet of several thousand AU pointing southwest. The morphology of this jet has been resolved by SINFONI observations (Nürnberger et al., in prep.) displaying three individual emission knots.

An optical spectrum of the bipolar nebula shows a variety of emission lines, changing their strength and profile with respect to the distance from the disk. The $\mathrm{H} \alpha$ and the Ca II $8498 \AA$ profiles from the SW lobe (Fig. 4) exhibit blue-shifted absorption indicative of accretion disk-driven outflows and a velocity dispersion of $\pm 500 \mathrm{~km} / \mathrm{s}$. The $\mathrm{H} \alpha$ profile is significantly different from the first spectrum obtained in 2003 (Chini et al. 2004) where a clear inverse P Cygni profile was visible.

\section{Sequential star formation}

The spatial distribution of stars in the M17 cluster strongly suggests a scenario of triggered star formation: Eight "naked" O-type stars (Cat. I) are located in the cluster center. The remaining massive objects discussed above (Cat. II and III) are situated southwest of this center toward the adjacent molecular cloud M 17 SW. Likewise, the fraction of NIR excess objects increases from a minimum of $7 \%$ in the eastern part to more than $50 \%$ in the southwestern region.

Even more interesting, among 109 objects with $K$-band spectra we find about $50 \%$ with $\mathrm{CO}$ band head absorption. Among these, 27 objects have X-ray emission and 20 show NIR excess; all are located in the southwestern area, B 273 being one of them. We suggest, that this is a population of intensely accreting luminous protostellar objects similar to IRC2c in Orion (Moreno et al. 1998) just formed within the dense environment of the molecular cloud.

In summary, the different evolutionary stages of massive objects, the high infrared excess frequency at the interface of the HII region and the molecular cloud and a new population of luminous $\left(L_{\mathrm{bol}} \sim 10^{4} \mathrm{~L}_{\odot}\right)$ objects with X-ray emission, often showing the $2.3 \mu \mathrm{m} \mathrm{CO}$ feature in absorption corroborate a picture of triggered star formation. 


\section{Conclusions}

On the basis of their $K$-band spectra, $X$-ray properties and the emission from their circumstellar environment we find that the massive objects in M17 can be divided into three categories:

- Cat I - There are eight major ionizing sources showing typical absorption line spectra of early-type O-type stars and $X$-ray emission; these objects are "naked" with respect to any circumstellar material.

- Cat II - This group of massive stars shows absorption line spectra typical for late $\mathrm{O}$ and early B-type stars and also exhibits $X$-ray emission. However, there is clear evidence for hot dust up to 1.000 AU from the star - seen as bright rims in the near-infrared and extended $(\sim 10.000 \mathrm{AU})$ warm dust in the mid-infrared. In addition, many sources are associated with (bipolar) HeI $1.083 \mu \mathrm{m}$ flows or filaments.

- Cat III - Probably the youngest group, this category comprises heavily reddened objects with emission line spectra and luminosities compatible with late $\mathrm{O}$ and early B-type stars; these object do not always show $X$-ray emission. Again there is evidence for bright near-infrared rims close to the star and mid-infrared emission from warm dust coming from a region of $\sim 4.000 \mathrm{AU}$.

The fan- or cometary-shaped $L$-band emission probably originates from hot dust grains the outer disk regions melted by the strong radiation field of the Cat I O-type stars. Often, these emission rims point toward the central mayor ionizing O4 binary. The extended MIR emitting material appears at various bands from 8 to $20 \mu \mathrm{m}$ - thus excluding pure PAH emission and suggesting the presence of warm dust. One-dimensional radiative transfer models have shown that the MIR emission of early B stars in M 17 can in principle be explained by warm $(\sim 100 \mathrm{~K})$ dust cocoons of several thousand AU in radius (Chini \& Krügel 1985). Likewise, the emission may originate from the surface of a warped disk directly heated by stellar photons.

The presence of both circumstellar dust and gaseous flows strongly supports the idea that the accretion is also working in stars as massive as O8. The global star formation in M 17 proceeds into the adjacent molecular cloud as witnessed by the various categories of massive stars, the new populations of NIR excess objects and CO absorption sources with X-ray emission.

\section{Acknowledgements}

This work was supported by the Nordrhein-Westfälische Akademie der Wissenschaften.

\section{References}

Chini, R., Elsässer, H., Neckel, Th. 1980, $A$ A 91, 186

Chini, R., Krügel, E. 1985, AA 164, 175

Chini, R., Wargau, W.F. 1998, AA 329, 161

Chini, R., Hoffmeister, V.H., Kimeswenger, S., Nielbock, M., Nürnberger, D., Schmidtobreick, L., Sterzik, M. 2004, Nature 429, 155

Hanson, M.M., Howarth, I.D., Conti, P.S. 1997, ApJ 489, 698

Felli, M., Churchwell, E., Massi, M. 1984, AA 136, 53

Moreno, J.-I., Yamashita, T., Hasegawa, T., Nakano, T. 1998, Nature 393, 340

Nielbock, M., Chini, R., Jütte, M., Manthey E. 2001, AA 377, 273

Kassis, M., Deutsch, L.K., Campbell, M.F., Hora, J.L., Fazio, G.G 2002, ApJ 124, 1636

Townsley, L. K., Broos, P. S., Chu, Y.-H., Feigelson, E. D., Garmire, G. P., Getman, K. V., \& Montmerle, T. 2003, Rev. Mex. de Astronomia y Astrofisica Conference Series, 15, 190 